\title{
Effect of Postural Changes on Baroreflex Sensitivity: A study on the EUROBAVAR data set
}

\author{
Younhee Choi \\ University of Rhode Island \\ Department of ECE \\ Kingston, RI 02881 \\ USA
}

\author{
Seok-Bum Ko \\ University of Saskatchewan \\ Department of EE \\ Saskatoon, SK S7N 5A9 \\ Canada
}

\begin{abstract}
The effect of changing posture from supine to standing on the heart rate variability was analyzed on the EUROBAVAR data set using power spectral analysis. Power spectral analysis has been commonly used to provide indices of autonomic cardiovascular modulation. Although the interpretation of power spectra is in dispute, it is generally agreed that the high-frequency (HF) power components represent the cardiac vagal modulation and the low-frequency (LF) power components are related to sympathetic activity. And then their ratio (LF/HF) is considered as an index of sympathovagal balance. In the supine lying position, relatively fast vagal activity plays a dominant role, whereas the upright standing position results in vagal inhibition and sympathetic predominance. Postural changing from supine to standing position showed the significant decrease in the indices of vagal influence on HRV; HF power in both absolute and normalized unit decreased and LF/HF increased. The baroreflex sensitivity values were estimated by time sequence method, $\alpha$-coefficients and transfer gains in LF and HF bands. There was a clear difference between BRS values in the supine and the standing positions with the averaged supine-to-standing BRS ratios of $2.35 \pm 0.4 ; 2.47 \pm 0.01$ of HF BRS ratios and $1.84 \pm 0.01$ of LF BRS ratios, $P<0.001$. Thus, the effect of changing positions to standing from lying can be concluded as a clear reduction in vagal influence on the heart rate variability.
\end{abstract}

Key-Words: Baroreflex sensitivity (BRS), Time sequence method, Power spectral analysis, Autonomic nervous system (ANS)

\section{Introduction}

Baroreflex sensitivity (BRS), which defines the relationship between a change in RR interval (RRI, the reciprocal of heart rate) to a given change in systolic blood pressure (SBP), has been considered as prognostic indicators of a number of cardiovascular diseases. Ever since the first measurement of BRS by vasoactive drug administration, several modern techniques have been developed to estimate BRS from the computer analysis of spontaneous fluctuations of cardiovascular variables obtained with noninvasive measuring equipments. Among the modern techniques for evaluating BRS, the time sequence method investigates the relationship between beat-to-beat time series of systolic blood pressure (SBP) and R-R interval, and the power spectral method analyzes them in the frequency domain $[1,2,3,4,5]$.

In addition, power spectral analysis of the heart rate variability (variance of R-R intervals) has been used in order to quantify the activity of the different autonomic nervous components. Although the interpretation is still not totally resolved, it is currently held that the power of high-frequency is related to the cardiac vagal modulation and the power ratio between low- and high-frequency bands is considered as a marker of sympathovagal activity $[2,5]$. A number of studies have been performed to investigate the differential modulation of parasympathetic and sympathetic nervous system outflow during postural change by estimating BRS using various methods. The main conclusion is that BRS is reduced on upright standing. As for the mechanism responsible for the reduced BRS, it is thought to involve parasympathetic nervous system withdrawal resulting in a reduction of available vagal nerve activity with which to regulate heart rate [16].

There has been an effort to speculate how fast the R-R intervals change in response to the variation in blood pressure by observing phase relationship between SBP and RRI during lower body negative pressure [15]. Time series data of SBP and RRI were searched for valid spontaneous barosequences (baroreflex sequences) in which SBP affected the current (lag 0), next (lag 1), or next following (lag 2) R-R 
interval. It is suggested that the lag of a baroreflex event is dependent on the length of the R-R interval.

Therefore, the objective of this study was to determine the effect of changing posture on vagal and sympathetic nerve activities. To do so, the power spectra of the heart rate variability regarding subject position was investigated and baroreflex sensitivity was estimated by various methods.

\section{Materials and Methods}

\subsection{Data Preparation}

The study was performed on the EUROBAVAR data set available from the internet provided by the Working Group on Blood Pressure and Heart Rate Variability of the European Society of Hypertension. The data were collected for a project originally aimed at comparing the BRS values obtained by different techniques performed on an identical set of data and also to evaluate the ability of each technique to detect the cases of baroreflex impairment. Refer to [4] or their website (www.cbi.dongnocchi.it/glossary/eurobavar.html) for the detailed demographic data of subjects. Briefly, the EUROBAVAR data set consists of 10 to 12 minute recordings from 21 subjects (17 women, 4 men) in both supine and standing positions using non-invasive monitoring devices. One subject was a diabetic patient with established cardiac autonomic neuropathy and one subject was a heart transplant recipient. For these two patients, very reduced heart rate variability is expected as a marker of baroreflex failure. The others were 12 normotensive outpatients and one untreated- and two treated-hypertensive subjects. The other four subjects were healthy volunteers.

Subject data on a beat by beat basis were used instead the entire curves of ECG and blood pressure wave. False or missed detections were manually replaced with the values calculated by linear interpolation between adjacent values. Any artifacts were corrected in the same way. Total 5 systolic blood pressure peaks from 3 subjects were corrected manually.

\subsection{BRS Estimates}

The time domain procedure begins with identifying sequences of three or more consecutive cardiac beats in which both systolic arterial blood pressure (SBP) and R-R interval (RRI) change in the same direction, either increase $(+\Delta \mathrm{RRI} /+\Delta \mathrm{SBP})$ or decrease ($\Delta \mathrm{RRI} /-\Delta \mathrm{SBP}$ ) [9]. A linear regression is applied to each individual sequence and the sequence is assumed to reflect the sensitivity of the cardiac baroreflex regulation only if the correlation coefficient exceeds 0.85 .
Individual BRS is obtained by averaging all the slopes identified as valid baroreflex sequence. In addition to the minimum correlation coefficient, there are several other parameters reported to affect the performance of BRS estimation by the time sequence methods [4]: three or more consecutive beats were used for validating a sequence and no restrictions were applied to the minimal changes in pressure or RRI and the minimal number of sequences for validating a spontaneous BRS estimate. In terms of the latency between SBP and RRI, 0-, 1-, and 2-beat(s) delay were examined to determine phase relationship between them corresponding postural change.

For spectral analysis, beat-to-beat RR intervals and systolic arterial pressures were then resampled at $4 \mathrm{~Hz}\left(T_{s}=0.25 \mathrm{sec}\right)$ to construct equidistantly spaced time series. Resampling was done by using cubic spline interpolation function. In order to eliminate the nonharmonic components in the very low frequency region, the dc component of signal was excluded in the calculation of power spectra. There are two different approaches to estimate BRS using spectral analysis: (1) an index, termed $\alpha$-coefficient, computes the square root ratio between R-R interval spectral power and systolic pressure spectral power at low- and highfrequencies

$$
\alpha \mathrm{LF}=\left.\sqrt{\frac{P_{R R I}}{P_{S B P}}}\right|_{L F}, \alpha \mathrm{HF}=\left.\sqrt{\frac{P_{R R I}}{P_{S B P}}}\right|_{H F}
$$

and (2) the transfer function gain uses R-R interval and systolic pressure cross-spectral magnitude in corresponding frequency bands.

$$
\operatorname{csLF}=\left.\frac{P_{R R I, S B P}}{P_{S B P}}\right|_{L F}, \operatorname{csHF}=\left.\frac{P_{R R I, S B P}}{P_{S B P}}\right|_{H F}
$$

Only bands in which a certain level of coherence $(>0.5)$ was reached were used to calculate the $\alpha$ coefficient or the transfer gain.

\subsection{Spectral Analysis of HRV}

Methods for the spectral estimates include nonparametric method based on the discrete Fourier transform and parametric method often referred to as autoregressive modelling (AR). The advantages and disadvantages of these procedures are well described in $[2,4]$. To avoid the basic disadvantage of AR modelling, the verification of the model order and of the suitability of the chosen model based on empirical knowledge, the power spectra were calculated by means of fast Fourier transformation.

In the power spectral analysis of short-term fluctuations of heart rate variability, three main components are distinguished as very low frequency (VLF) 
components, low-frequency (LF) components and high-frequency (HF) components. The ranges for each frequency bands are different for studies, but, in this study, VHF, LF, and HF powers were defined in the range of $0.01-0.04 \mathrm{~Hz}, 0.04-0.15 \mathrm{~Hz}$, and $0.15-0.4$ $\mathrm{Hz}$, respectively. The area of spectral peaks within the whole range of 0.01 to $0.4 \mathrm{~Hz}$ was defined as total power. The power of the LF and HF components is measured in both absolute values $\left(\mathrm{ms}^{2} / \mathrm{Hz}\right)$ and normalized units (n.u.), which represent the relative value of each power component in proportion to the total power minus the VLF component. By measuring LF and HF power in normalized units, the effect of the changes in total power on each frequency bands can be minimized. While there is general agreement that the efferent vagal activity is a major contributor to the HF component, the mechanisms underlying the LF component are more controversial. With an assumption that low- and high-frequency power components, especially in normalized units, represent sympathetic and vagal cardiac drive, respectively, their ratio $(\mathrm{LF} / \mathrm{HF})$ is considered as an index of sympathovagal balance.

\subsection{Statistical Analysis}

A paired $t$-test was used to compare mean SBP, mean $\mathrm{R}-\mathrm{R}$ intervals, BRS values, and the various measurements of power in interesting frequency bands between supine and standing positions. Also BRS values measured by the sequence method, the spectral analysis in the LF band, and the spectral analysis in the HF band were compared. Data were presented as means $\pm \mathrm{SD}$, and differences were considered statistically significant when $P<0.05$.

\section{Results}

The R-R interval decreased significantly from supine lying to standing $(0.88 \pm 0.17 \mathrm{~ms}$ to $0.75 \pm 0.14 \mathrm{~ms}$, $P<0.001$ ) while the systolic blood pressure was not changed appreciably from supine $(121 \pm 18.6 \mathrm{mmHg})$ to standing $(121.2 \pm 21.4 \mathrm{mmHg})$. In other words, considering the definition of baroreflex sensitivity (or gain), the R-R interval reflex responses to blood pressure changes $[\mathrm{s} / \mathrm{mmHg}$, diminished baroreflexmediated cardiac vagal modulation in standing position helped maintain the systolic blood pressure at a certain level by adjusting the R-R interval. Statistics along with BRS estimates by various techniques were summarized in Table 1.

The time sequence method was not able to provide BRS values for one subject (A003) in the supine position and for one subject (B005) in the standing
Table 1: Statistics and BRS estimates

\begin{tabular}{c||ccc}
\hline & Supine & Standing & $P$ \\
\hline SBP & $121 \pm 18.6$ & $121.2 \pm 21.4$ & n.s. \\
DBP & $61.6 \pm 11.7$ & $67.9 \pm 11.7$ & $<0.005$ \\
RRI & $0.88 \pm 0.17$ & $0.75 \pm 0.14$ & $<0.005$ \\
\hline SEQ & $14.9 \pm 10.6 \dagger$ & $7.1 \pm 4.2 \dagger$ & $<0.005$ \\
$\alpha$ LF & $12.37 \pm 12.33$ & $6.65 \pm 4.51$ & $<0.005$ \\
$\alpha H F$ & $18.94 \pm 16.59$ & $7.72 \pm 5.893$ & $<0.005$ \\
csLF & $10.6 \pm 10.81$ & $5.73 \pm 3.99$ & $<0.001$ \\
csHF & $16.35 \pm 14.26$ & $6.61 \pm 4.92$ & $<0.005$ \\
\hline
\end{tabular}

All values are mean \pm SD. SBP: systolic blood pressure [mmHg], DBP: diastolic blood pressure [mmHg], RRI: R-R interval [sec], SEQ, $\alpha \mathrm{LF}, \alpha \mathrm{HF}$, csLF, csHF: baroreflex sensitivity computed by time sequence method, the $\alpha$-coefficients and the transfer gains in low- and highfrequency bands, respectively $[\mathrm{ms} / \mathrm{mmHg}]$. n.s.: not significant. $\dagger$ The results were from 20 subject data excluding one subject with no valid BRS sequence.

position. For one subject (B004) in the supine position, only one valid BRS sequence was identified. The smaller BRS values in the supine position were accounted for by subject B005 and B010 who, with the results from spectral analysis, were considered as patients with evident baroreflex failure. There was a clear difference between BRS values for supine and standing; BRS values were higher when the subject was in the supine position. The differences were significant $(P<0.005)$ for both time sequence method and frequency domain analysis methods. On the other hand, BRS estimates using HF components indicated higher values than LF analysis. The averaged supineto-standing BRS ratio was $2.35 \pm 0.4$. Of note, the ratio between HF BRS estimates in supine and standing was greater than the ratio between LF BRS estimates.

It is consistent with [4] that BRS estimates derived from spectral analysis ( $\alpha$ LF-csLF and $\alpha \mathrm{HF}$ csHF, respectively, in low- and high-frequency bands) were highly correlated $(r>0.99)$ for both supine and standing positions. BRS estimates using HF bands were highly correlated with the BRS obtained by time sequence method for both supine and standing positions. The correlation coefficients between methods were presented in Table 2. Data for subjects, for whom no BRS value was estimated, were excluded.

As for the impact of subject position on heart rate variability, the changes in total power and LF power in absolute value were not significant. In contrast, HF power in both absolute value and normalized unit changed significantly according to subject position. The normalized HF power which can be interpreted as a maker of cardiac vagal activity de- 
Table 2: Correlation coefficients between methods

\begin{tabular}{|c|c|c|c|}
\hline & \multicolumn{2}{|c|}{ Supine } & Standing \\
\hline & SEQ $\alpha$ LF & $\alpha \mathrm{HF} \operatorname{csLF}$ & SEQ $\alpha$ LF $\alpha \mathrm{HF}$ csLF \\
\hline$\alpha \mathrm{LF}$ & 0.81 & & 0.78 \\
\hline$\alpha \mathrm{HF}$ & $0.95 \dagger 0.93 \ddagger$ & & $0.94 \dagger 0.7$ \\
\hline $\operatorname{csLF}$ & $0.8 \dagger$ & $0.87 \ddagger$ & $\begin{array}{llll}0.75 & 1 \ddagger & 0.68 \dagger\end{array}$ \\
\hline $\mathrm{csHF}$ & $0.960 .88 \ddagger$ & $1 \ddagger 0.87 \ddagger$ & $\begin{array}{llll}0.93 & 0.71 & 1 \ddagger & 0.69\end{array}$ \\
\hline
\end{tabular}

The results were from 19 subject data excluding subjects A003 and B005, for whom time sequence method failed to provide BRS estimation.

$\dagger P<0.05, \ddagger P<0.005$.

creased from 49.2 n.u. in lying to 36.8 n.u. in standing $(P<0.005)$. The power ratio $(\mathrm{LF} / \mathrm{HF})$ increased $(1.4$ to $2.65, P<0.005)$ in standing position. Power spectral analysis of HRV indices in supine and standing were listed in Table 3.

Several efforts to use spectral analysis as a clinical tool include the prognosis of heart transplant recipients, diabetic anatomic neuropathy, and hypertension $[5,9]$. There is more agreement among studies regarding the use of power spectral techniques in diabetic neuropathy. As described in [5], diabetic neuropathy can be diagnosed by investigating the power spectra of HRV if following abnormalities were found : (1) reduced power in all spectral bands, (2) failure to increase LF on standing, and (3) abnormally reduced total power with unchanged LF/HF ratio. Subject B005 and $\mathrm{B} 010$ were suspected as the patients with baroreflex impairment. Moreover, the smallest BRS values were obtained for B005 and B010 in both positions by all methods except B005 in standing position by time sequence method for whom no valid BRS sequence was identified.

The number of valid barosequences per 512 cardiac beats in supine and standing was illustrated in

Table 3: Power spectral analysis of HRV indices

\begin{tabular}{c||ccc}
\hline & Supine & Standing & $P$ \\
\hline Total power & $801 \pm 776$ & $676 \pm 935$ & n.s. \\
LF & $291 \pm 278$ & $280 \pm 400$ & n.s. \\
HF & $320 \pm 459$ & $121 \pm 131$ & $<0.05$ \\
nLF & $50.8 \pm 19.7$ & $63.2 \pm 21.7$ & $<0.005$ \\
nHF & $49.2 \pm 19.7$ & $36.8 \pm 21.7$ & $<0.005$ \\
LF/HF & $1.4 \pm 1.1$ & $2.65 \pm 2$ & $<0.005$ \\
\hline
\end{tabular}

All values are mean \pm SD. LF, HF: absolute power in low- and high-frequency bands $\left[10^{3} \mathrm{~ms}^{2}\right]$, nLF, nHF: normalized power in low- and high-frequency bands [n.u.], $\mathrm{LF} / \mathrm{HF}$ : ratio between power in low- and high-frequency bands. n.s.: not significant.

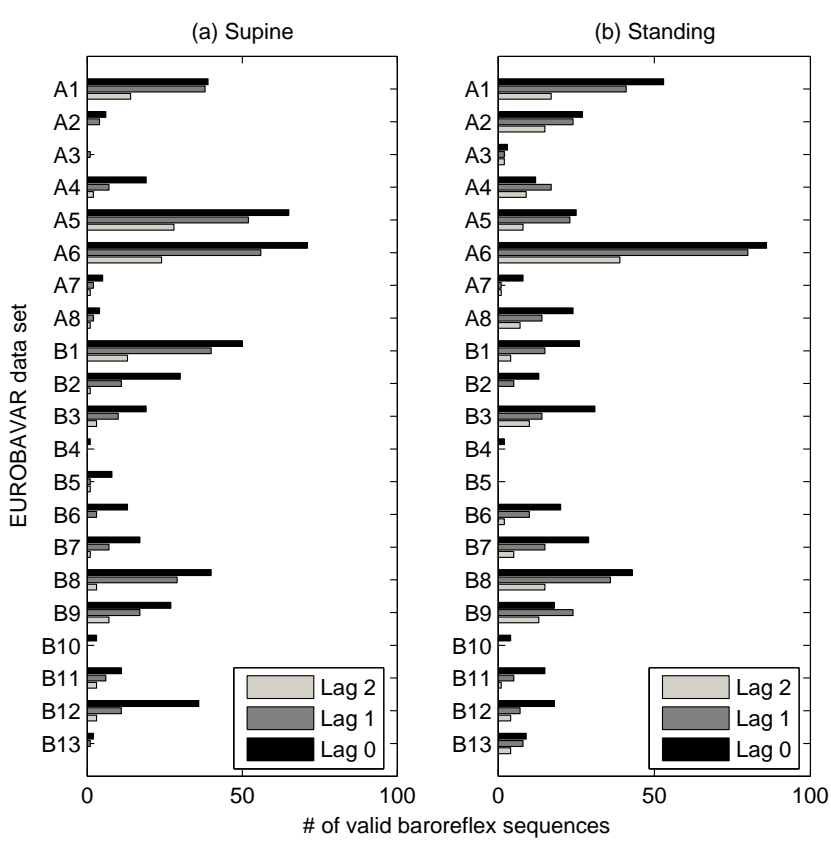

Figure 1: Number of valid baroreflex sequences per 512 heartbeats for each subjects when they are (a) supine lying and (b) standing.

Figure 1. As investigated in [15], beat-by-beat impact of systolic blood pressure on R-R interval is dependent on the length of the R-R interval. With decreased mean RRI $(0.88 \pm 0.17$ to $0.75 \pm 0.14 \mathrm{sec}$, $P<0.005$ ), there was no significant change in terms of the number of barorsequences upon standing in all three cases, 0-, 1-, and 2-beat(s) delay. However, the number of barosequences was significantly different among the choices of delay; 22.2 \pm 21.1 lag 0 sequences, $15.7 \pm 18.0$ lag 1 sequences, and $7.0 \pm 8.8$ lag 2 sequences in supine and $22.2 \pm 19.9$ lag 0 sequences, $18.9 \pm 18.8 \mathrm{lag} 1$ sequences, and 9.2 $\pm 9.3 \mathrm{lag}$ 2 sequences in standing $(P<0.005)$. As shown in Figure 2, In the supine, the BRS estimates with lag 0 sequences were different from the BRS estimates with lag 1 or lag 2 sequences $(15.2 \pm 11.2,13.0 \pm 7.0$, $12.1 \pm 6.6 \mathrm{~ms} / \mathrm{mmHg}, P<0.05, P<0.05$, respectively) while, in the standing, the BRS estimates with lag 0 sequences were different from the BRS estimates with lag 1 sequences $(7.2 \pm 4.1,6.5 \pm 3.5 \mathrm{~ms} / \mathrm{mmHg}$, $P<0.05$, respectively).

\section{Conclusion}

In the standing position the absolute values of $\mathrm{HF}$ power, normalized LF and HF power, and the ratios between LF and HF (LF/HF) are significantly different from the values measured when subjects were in the supine position. These effects of changing posi- 


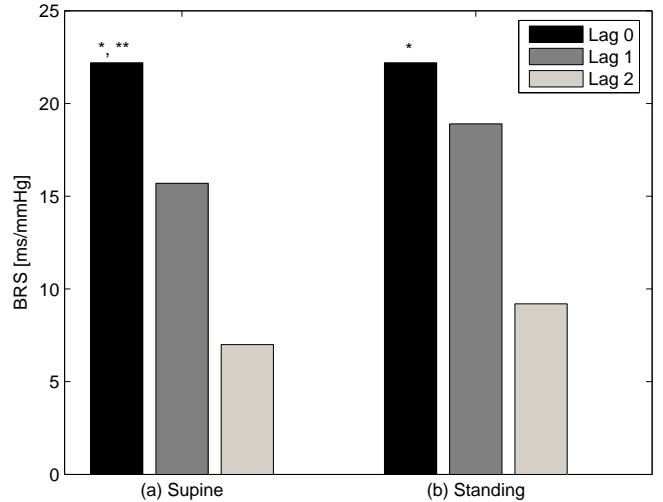

Figure 2: BRS estimates with three choices of delay; lag 0 , lag 1 , lag 2 sequences. * Significantly different from BRS estimates with lag 1 sequences, $* *$ significantly different from BRS estimates with lag 1 sequences. All $P<0.05$.

tions to standing from supine lying can be concluded as a clear reduction in vagal influence on the heart rate variability.

Six subjects out of total 21 showed increased normalized HF power when they change positions from supine lying into standing, which is the apposite of expectation; the vagal effect is depressed when subject is standing, thus normalized HF power decreased. But, it is notable that the average of increases in supine-tostanding normalized high-frequency power $(+\Delta \mathrm{nHF}$ : 10.4) was relatively small compared to the average of decreases ( $-\Delta \mathrm{nHF}: 22.3)$. Likewise, the averaged decrease in $\mathrm{LF} / \mathrm{HF}(-\Delta \mathrm{LF} / \mathrm{HF})$ was 0.65 and the averaged increase $(+\Delta \mathrm{LF} / \mathrm{HF})$ was 2.06 . It may be explained by the speculation that the heart rate variability arise not only from fluctuations in central neural activity but also from extra cardiovascular inputs such as mechanical and/or neural effects of respiration [13]. Also, as pointed out in [8], the heart rate variability data usually differed among measurements over a time span (intra-individual variability), especially they differed remarkably among successive day measurements, maybe it is not desirable to draw any conclusions from data set of single measurements.

As defined, the time sequence method approach can be affected by the number of sequences used to determine the average regression slope and the number of sequences is dependent on the choice of the beat delay between affecting blood pressure change and responding $\mathrm{R}-\mathrm{R}$ interval change. It has become standard practice to apply a lag of one beat for validating the baroreflex sequences $[15,16]$. The study on healthy young subjects [12] revealed that the effect of changes in SBP is observed on the immediate R-R interval (lag 0) if the R-R interval is $>775 \mathrm{~ms}$ but that it is observed on the next beats (lag 1 or lag 2) for shorter R-R intervals. 17 subjects out of 21 subjects fall in that range of relatively longer R-R interval in supine position, whereas only 9 subjects are applicable in standing position due to the reduced mean R$\mathrm{R}$ interval. As shown in Figure 2, not only barosequences without delay but a non-negligible number of barosequences with lags 1 and 2 are also present. Even when the R-R interval is sufficiently long that SBP affected the current R-R interval through arterial baroreflex response mediated by vagal modulation, the changes in SBP also affected the subsequent beats by sympathetic nerve activity and/or other nonbaroreflex mechanisms. It is consistent with the observation in [10] that there was a trend toward a increase in sequences with lag of 1 and 2 beat(s). However, for 13 out of 21 subjects used in this study, the number of lag 0 sequences increased. The discrepancy might be caused by their criteria for selecting subjects; only subjects with a resting R-R interval $>900 \mathrm{~ms}$ and a reduction in $\mathrm{R}-\mathrm{R}$ interval to $<850 \mathrm{~ms}$ when the lower body negative pressure of $-40 \mathrm{mmHg}$, or greater, was applied. Only 2 subjects from EUROBAVAR data set meet these criteria.

In conclusion, regardless of the mechanisms involved, the present data are consistent with other studies including $[7,8,10,14,16]$ that spontaneous vagal influence is reduced in the standing. As pointed out in [4], the setting of parameters should be optimized to estimate the baroreflex sensitivity. This study supported this by showing that BRS estimates were significantly different with the choice of $0-, 1-$, or 2beat(s) delay between affecting blood pressure change and responding $\mathrm{R}-\mathrm{R}$ interval change.

\section{References:}

[1] Di Rienzo M, Castiglioni P, Mancia G, Pedotti A, and Parati G: Advancements in estimating baroreflex function. IEEE Eng Med Biol Mag 20(2): 25-32, 2001

[2] Parati G, Saul JP, Di Rienzo M, and Mancia G: Spectral analysis of blood pressure and heart rate variability in evaluating cardiovascular regulation: a critical appraisal. Hypertension 25: 12761286, 1995

[3] Westerhof BE, Gisolf J, Stok WJ, Wesseling $\mathrm{KH}$, and Karemaker JM: Time domain crosscorrelation baroreflex sensitivity: performance on the Eurobavar data set. J Hypertensions 22: 1371-1380, 2004

[4] Laude D, Elghozi JL, Girard A, Bellard E, Bouhaddi M, Castiglioni $\mathrm{P}$ et al:: Comparison 
of various techniques used to estimate spontaneous baroreflex sensitivity (the EuroBaVar study). Am J Physiol Regul Integr Comp Physiol 286: R226-R231, 2004

[5] Task Force of the European Society of Cardiology and the North American Society of Pacing and Electrophysioloy: Heart rate variability. Standards of measurement, physiological interpretation, and clinical use. Circulation 93: 10431065, 1996

[6] Lipman RD, Salisbury JK, and Taylor JA: Spontaneous indices are inconsistent with arterial baroreflex gain. Hypertension 42: 481-487, 2003

[7] Kuo CD and Chen GY: Comparison of three recumbent positions on vagal and sympathetic modulation using spectral heart rate variability in patients with coronary artery disease. Am J Cardiol 81: 392-396, 1998

[8] Kalisnik JM, Avbelj V, Trobec R, and Gersak B: Imaging of power spectral heart rate variability regarding subject position. Eur J Physiol 442: R142-R144, 2001

[9] Persson PB: Spectrum analysis of cardiovascular time series. Am J Physiol Regulatory Integrative Comp Physiol 273: R1201-R1210, 1997

[10] Blaber AP, Yamamoto Y, and Hughson RL: Change in phase relatioship between SBP and $\mathrm{R}-\mathrm{R}$ interval during lower body negative pressure. Am J Physiol 268 (Heart Circ Physiol 37) H1688-H1693, 1995

[11] Di Rienzo M, Parati G, Castiglioni P, Tordi R, Mancia G, and Pedotti A: Baroreflex effectiveness index: an additional measure of baroreflex control of heart rate in daily life. Am J Physiol Regul Integr Comp Physiol 280: R744-R751, 2001

[12] Pickering TG and Davies J: Estimation of the conduction time of the baroreceptor-cardiac refelx in man. Cardiovasc Res 7: 213-219, 1973

[13] Stauss H: Heart rate variability. Am J Physiol Regul Integr Comp Physiol 285: R927-R931, 2003

[14] O'Leary DD, Kimmerly DS, Cechetto AD, and Shoemaker JK: Differential effect of head-up tilt on cardiovagal and sympathetic baroreflex sensitivity in humans. Experimental Physiology 88.6: 769-774, 2003

[15] Hughson RL, Quintin L, Annat G, Yamamoto $\mathrm{Y}$, and Gharib C: Spontaneous baroreflex by sequence and power spectral methods in humans. Clin Physiol 13: 663-676, 1993
[16] Steptoe A and Vogele C: Cardiac baroreflex function during postural change assessed using non-invasive spontaneous sequence analysis in young men. Cardiovasc Res 24(8): 627-32, 1990 